\title{
CONTRIBUTIONS OF THE USNO TO THE OPTICAL
}

\author{
REFERENCE FRAME
}

\author{
THOMAS CORBIN \\ Astrometry Department, U.S. Naval Observatory \\ Washington DC, USA
}

\section{Introduction}

A good, working definition of what is required in a celestial reference frame is that it must provide observable fiducial points on the Celestial Sphere with internally consistent positions that are referred to coordinate axes of known direction. In reality, this statement gives the goals in the reverse order from that in which each must be achieved, the definition of the axes, or zero points of the system give orientation to the observationally defined set of primary objects whose coordinate relation to each other must give the frame rigidity. Finally, the primary objects are generally too sparse to define the frame within areas of less than tens of square degrees, and so additional objects must be related to the frame to increase the density. This last step is required to make the frame useful for most observational applications.

The U.S. Naval Observatory (USNO) has been an active contributor to the optical reference frame for all of this century. Its current and upcoming programs both continue in this vein and encompass the new technologies required for the rapidly evolving definition of the optical frame.

\section{Reference Frame Orientation and Internal Consistency}

\subsection{COORDINATE AXES}

Although the dynamical system, defined by the motions of solar system objects, has provided the zero points for the optical frames defined by the fundamental star catalogs up to, and including, the FK5 (Fricke et al., 1988, 1991), the next orientation will be defined by VLBI radio observations of extragalactic sources. The first realization of this new frame was titled the

I. M. Wytrzyszczak, J. H. Lieske and R. A. Feldman (eds.),

Dynamics and Astrometry of Natural and Artificial Celestial Bodies, 453, 1997.

(C) 1997 Kluwer Academic Publishers. Printed in the Netherlands. 
Radio Optical Reference Frame (RORF) and was produced by a collaboration of the USNO, Geosciences Laboratory, NASA and JPL (Johnston et $a l ., 1995)$. The axes were brought into coincidence with the IERS system and are consistent with those of the FK5. The RORF contains 424 defining sources, and the individual sources give an internal consistency of 1 milliarcsecond (mas) north of the Celestial Equator and 3 mas or better in the south.

The final definition of the extragalactic frame has been made under the IAU Working Group on Reference Frames. The resulting frame was compiled from the list of candidate sources adopted in IAU Resolution B5 (1994) and is be known as the International Celestial Reference Frame (ICRF) (Morrison, 1995).

The ICRF will be the standard reference for celestial coordinates, thereby replacing the FK5. The observations and data reductions accomplished for the RORF constitute an important part of the ICRF. Furthermore, the USNO has continued to be very active in the continuing improvement of the RORF. This has included scheduling regular observations at 12 participating VLBI stations and collecting, correlating and reducing data to improve the frame. The positions of all 610 sources have now been brought to a precision of 1 mas, or better, with 197 of the best-observed below the 0.2 mas level. At this level some $50 \%$ of the sources show change, and a very active program is underway to map the structure of the sources and monitor them for change. At this time imaging of 194 of the sources has been completed.

The USNO is also paying particular attention to relating the positions and motions of as many radio stars as possible to the ICRF. This important class of objects is the only one that is directly observable by the primary instruments that define the radio and optical frames. Since these objects are weak sources at radio wavelengths, special approaches, such as phasereferencing, must be employed. This work is underway and the goal is to increase the number that can be used to link the radio and optical frames. The link of Hipparcos to the extragalactic frame employed 19 radio stars (Kovalevsky et al., 1997), and it is hoped to raise this number to 24 .

\subsection{OPTICAL REALIZATION}

The Hipparcos observational frame, rotated to that of the ICRF, will mark the beginning of the optical reference frame referenced to coordinate axes defined by extragalactic objects. However, while the ICRF objects are stationary at the 1 mas level, the Hipparcos stars are not. When Hipparcos is made generally available next year, it will be some six years past its cen- 
tral epoch of 1991.25. To prevent deterioration of the system due to proper motion, high-precision observations must be made on a continuing basis.

\subsubsection{The Navy Prototype Optical Interferometer (NPOI)}

The NPOI in Flagstaff, Arizona (Hutter et al., 1995), the result of collaboration between the USNO and Naval Research Laboratory, has been under construction for several years and is now about to begin its first observing program. This program will consist of about 1000 stars for declination $>-20^{\circ}$. The limiting magnitude will be $m_{v} \approx 10$, and this will be achieved with four $0.5 \mathrm{~m}$ siderostats giving three independent baselines of 18 to $35 \mathrm{~m}$. The metrology of the system will be at the $100 \mathrm{~nm}$ level. The result of this configuration will be wide-angle astrometry at the milliarcsecond level. The observed, instrumental frame will be aligned with the ICRF through the inclusion of all radio stars that may be successfully observed with VLBI techniques. The resulting catalog, with an epoch of 1997, will provide the first observational check on both the frame of Hipparcos and the individual positions and motions of some of its component stars.

The NPOI also has imaging capabilities using six moveable, imaging siderostats (Hutter, 1995) operating on baselines up to $435 \mathrm{~m}$. These will give resolutions of 0.2 mas and better. The power of a preliminary version of this system was demonstrated by a clear series of images showing the orbital motion of the 6 mas binary $\zeta^{1}$ Ursae Majoris.

\subsubsection{Astrographic Link}

The USNO, in collaboration with the Hamburger Sternwarte, has been engaged in making astrographic observations to link Hipparcos with the ICRF. Based initially on the International Reference Stars (IRS) (Corbin and Urban, 1990) and now on Hipparcos, astrographic plates and CCD frames are used to map successively fainter and smaller areas around the optical counterparts of the ICRF objects. Finally the counterparts themselves are imaged and measured against the reference stars (Zacharias et al., 1995).

The instruments employed are: the Hamburg Astrograph and the USNO Twin Astrograph, reaching magnitudes 12-14; the USNO CCD Astrograph (UCA), reaching magnitudes 15-16; and the Kitt Peak National Observatory (KPNO) $0.9-\mathrm{m}$ and the Cerro Tololo Inter-American Observatory (CTIO) $0.9-\mathrm{m}$, reaching magnitudes 18-20. CCDs are used with the UCA and the two $0.9-\mathrm{m}$ instruments. The small fields of the CCDs allow astrometric measures to be made at the flat field centers of the two $0.9-\mathrm{m}$ telescopes. However, in order to get a sufficient number of reference stars at each stage, the fields must be overlapped and a mosaic process of reduc- 
tion employed. Zacharias generally uses $2 \times 2$ or $3 \times 3$ frame mosaics with overlapping frames to tie the mosaics together.

Thus, far 437 fields have been observed with 130 completely reduced. Field distortions in the $0.9-\mathrm{m}$ instruments currently set the limit for the accuracy of the link. Using Hipparcos data, Zacharias estimates that the link for each ICRF field will be accurate to 10-20 mas, which will give a link accuracy for the Hipparcos-ICRF orientation at the 1-mas level for the whole ensemble.

\section{Reference Frame Density}

The Hipparcos catalog will have an average density of 2.9 stars per square degree. However, since the Hipparcos list is not uniformly distributed over the sky, this number drops to 1.7 in some areas at high galactic latitudes. These densities are not high enough for many astrometric applications, especially those involving the small fields of CCD's. Tycho has a much higher density, about 24 stars per square degree, but beyond magnitude 11.5 both the number of stars and the positional quality decline. Since many observing programs now require both fainter and more dense reference stars than even Tycho can offer, additional observing programs are needed to raise the density of the optical reference frame. The USNO is involved with several programs that address this issue.

\subsection{THE TWIN ASTROGRAPH CATALOG (TAC)}

From 1977 to 1985 astrographic plates were taken with the USNO 8" Dual Astrograph. This instrument consisted of two telescopes, one with a "blue" (bandpass 410 to $486 \mathrm{~nm}$ ) lens and the other with a "yellow" lens (508 to $578 \mathrm{~nm}$ ). Each field was photographed simultaneously with the blue and yellow lenses in a twofold overlap manner. Thus, each field was covered in a fourfold manner. In all 5180 plates north of $-18^{\circ}$ were taken.

A first version of the TAC has been produced from the 4912 plates measured thus far and reduced with the IRS (Zacharias et al., 1996). This catalog contains 705679 stars to magnitude 11.5, about 30 stars/sq. degree, with an average epoch of 1982.5 . The positional accuracy is 86 and 91 mas in right ascension and declination, respectively. The positions are being combined with new reductions of the Astrographic Catalogue to give proper motions of about 3 mas/yr accuracy. The catalog will be available by the end of 1996.

Future work will include measurement of the remaining plates, $20^{h}$ through $6^{h}$ of RA for the plates centered at $-15^{\circ}$ to $-9^{\circ}$ and $+65^{\circ}$ to $+90^{\circ}$. New reductions will also be made when Hipparcos becomes available, and recent work indicates that the errors of the positions will drop below 60 mas at that point. 


\subsection{SLOAN DIGITAL SKY SURVEY (SDSS)}

The USNO initially became interested in the SDSS because it afforded an opportunity to gain experience with CCD arrays for possible astrometric applications. The USNO is now a full partner in the data processing consortium and is responsible for the project's astrometric reductions.

The goal of the SDSS is to survey the sky within an ellipse $55^{\circ}$ to $65^{\circ}$ of the North Galactic Pole in five bandpasses (Gunn, 1995). The survey will go very deep, to red magnitude 23 , and will result in a very extensive database of magnitudes, colors, and positions of galaxies, quasars and stars. Spectroscopy will also be done for galaxies, quasars and interesting stars.

The original astrometric goals of the project were mainly concerned with sufficient positional accuracy (200 mas) to identify objects and to prepare templates for fiber optic lines to do the spectroscopy. When the USNO personnel became involved, however, it became clear to them that the project has the potential to do much better.

The SDSS will involve a $2.5 \mathrm{~m}$, dedicated telescope employing an array of 54 CCD's, 30 of which will be used for the photometry - the others being employed for astrometry and focus. The observations will be made in a scanning mode with the telescope being driven along great circles.

The ranges of the CCD chips will be magnitudes 8 to 15 for the astrometric and 14 through 23 for the photometric. Thus, the astrometric chips will provide the reference stars and will tie the results to the optical frame through Hipparcos and Tycho. The target is to obtain positions in the 50 to 100 mas range to the $18^{\text {th }}$ magnitude for at least 5000000 stars. If these goals are realized, the project will help point the way to all-sky surveys that will extend the optical frame to very faint magnitudes.

\subsection{USNO CCD ASTROGRAPH (UCA)}

In recent years there has been an increasing need for reference stars reaching the 14 to 15 magnitude range. Until the appearance of Hipparcos, Tycho and TAC the existing surveys only extended to magnitude 11 , and even these new surveys do not go much past that limit.

The USNO originally envisioned a photographic survey with the $8^{\prime \prime}$ Twin Astrograph. However with the advances in CCD technology and the development of a new, high-quality lens system (bandpass 550 to $710 \mathrm{~nm}$ ) for the telescope, it was felt that a single-lens instrument with a CCD camera would give the best results.

The instrument is currently under development (Zacharias, 1997) and will employ a $4096 \times 4096 \mathrm{CCD}$ that will yield a $1^{\circ}$ field. The telescope will operate in an automatic mode that will take overlapping frames, each of which will be reduced with Tycho stars. The use of a grating will permit 
precise measurement of the Hipparcos stars for the final reduction of the data with block adjustment techniques. This will give a solution independent of the Tycho stars.

It is planned to begin the program in the Southern Hemisphere. The limiting magnitude would be $R=15.5$. Based on current experience with the instrument mounted in Washington and using a $1536 \times 1024$ chip, the expected accuracies are 20 mas for stars in the range $R=6$ to 14 and 30 mas at $\mathrm{R}=15.5$; with errors increasing more rapidly beyond that.

Thus, the USNO CCD Astrograph is capable of producing Tycho-quality positions for a very large number of stars. This extension of the optical frame would greatly benefit a wide range observational programs that currently are unable to obtain suitable reference stars for their reduction needs.

\subsection{THE PRECISION MEASURING MACHINE (PMM)}

The PMM operates at the USNO Flagstaff Station where it was designed and had its software developed (Pier and Monet, 1993). The machine employs two CCD cameras, each of $1312 \times 1033$ pixels. The pixels are 6.8 microns each and the digitization is 10 megapixels/second. The PMM operates automatically with a precision of 0.2 pixel and can detect and measure 1000000 objects on a photographic plate in 60 minutes. The PMM was designed to measure the plates of the Palomar Sky Survey and therefore needed to have great speed while maintaining accuracy.

The first epoch Palomar plates, POSS-I, were taken from 1950 to 1958 in two bandpasses (350-540 $\mathrm{nm}$ and $620-670 \mathrm{~nm})$ and cover the sky north of $-30^{\circ}$ in 937 fields. The second epoch, POSS-II, began in 1987 and is expected to finish in 1997. POSS-II was done in three bandpasses, 350$500 \mathrm{~nm}, 610-690 \mathrm{~nm}$ and $730-900 \mathrm{~nm}$, covering the sky north of $0^{\circ}$ in 894 fields. The effective magnitude limit of both surveys is 20 .

The measuring of POSS-I is complete, and the POSS-II plates are measured as they are taken. The POSS-II will contain roughly 500000000 objects and the catalog accuracy is expected to be 150 mas. Since the POSS-I accuracies will be somewhat lower, about 200 mas, the proper motions resulting from combining the two surveys will be in the 6 to 7 mas/year range. The POSS I and II will be given new reductions when Tycho becomes available to tie the positions and motions to the Hipparcos frame.

The catalog from POSS-I/II, in addition to providing positions and motions of an enormous number of stars, will contain magnitudes, colors and identifications of non-stellar objects (Monet, 1996). This tremendous database will thus not only provide reference stars at extremely high densities, but will also serve as a valuable photometric resource for a wide range of applications. 


\subsection{THE ASTROGRAPHIC CATALOGUE (AC)}

The USNO has been making new reductions of the zones of the Astrographic Catalogue with the intent of providing accurate positions at at early epoch for the purpose of determining proper motions (Urban and Corbin, 1996). The work on the AC is described in detail by Urban elsewhere in these proceedings. It is therefore sufficient to say that the $\mathrm{AC}$ is a valuable resource with an average epoch of 1905 and positions good to about 200 mas after rigorous reduction. Using the AC in combination with the results from Tycho, the UCA or the SDSS, proper motions accurate to 3 mas/yr can be obtained for stars down to the $12^{\text {th }}$ magnitude.

\section{Reference Frame Clarification}

It has been traditional in transit circle catalogs and astrograph surveys to identify stars that are not single and to indicate to what the observed position refers (usually center of light, A-component, or center of mass). However, the center of light is not necessarily the same in two different instruments, and this often has led to errors when combining catalogs to compute proper motions. Observing one companion also had its problems because uncorrected orbital motion would affect the observed positions at different epochs and introduce errors into the proper motions.

As astrometry pushes toward ever higher accuracies, the problem of complex objects becomes more serious. The latest manifestation of this has been the "problem stars" in the Hipparcos program. In a like manner, the high accuracies required to link the optical and extragalactic frames means that there must be information on the centers of emission at both wavelengths. The USNO is taking an active role in dealing with these two problems. The result is that imaging is becoming increasingly important in the USNO astrometric programs.

\subsection{THE WASHINGTON DOUBLE STAR CATALOG (WDS)}

The WDS is the world's primary database for double and multiple stars. The data are maintained as a resource for both individual observations and as a double star catalog. The 1996.0 version (Worley and Douglass, 1993) of the WDS has just been completed and contains 448100 observations of 78100 systems. In addition, a new catalog of orbits is in preparation.

Since wide-angle astrometric instruments have generally not had high image resolution capabilities, separate series of observations have been necessary to deal with the problem of multiple stars. The USNO has had, for many years, an active program of double star observations. This program was conducted with a visual micrometer on the 66-cm refractor until 
1990 , at which time a speckle interferometry camera was installed. Since then some 7000 speckle observations have been made. This high rate of output has been possible because the $66 \mathrm{~cm}$ refractor is dedicated to the double star observing program. The USNO program currently can observe pairs of stars up to $5^{\prime \prime}$ of separation and with a magnitude difference of 3.5. The limiting magnitude is 10 , and for stars of equal brightness the limiting separation is 250 mas.

There are two extensions of the USNO observations for the WDS currently underway. First, there are plans to extend the speckle camera program to larger instruments. A $1.5-\mathrm{m}$ telescope would extend the magnitude limit to 13 , but the resolution would be about the same since the focal lengths for these telescopes are about the same as that of the $66-\mathrm{cm}$ refractor. A 3.5-m instrument, however, should give resolution of 50 mas and a limiting magnitude of 15 . The second extension would involve the NPOI. As mentioned above, this instrument is capable of resolving very close systems. Its imaging capabilities will allow it to survey stars too closely separated for the speckle camera.

Thus, the USNO observations for the WDS will span separations from 5 arcsec to 0.1 mas. The main problem is the sheer number of stars that need surveying. Both the speckle camera and the NPOI are designed to give accurate measures on a star-by-star basis, and neither is well-suited for surveys on a large scale. Another problem is that the NPOI cannot work with stars fainter than $10^{\text {th }}$ magnitude. Even the speckle camera on a $3.5-\mathrm{m}$ telescope only reaches the middle of the magnitude range of programs like the POSS-I/II or the SDSS. Since roughly $50 \%$ of the stars appear to be double or multiple systems, they will present a problem to high accuracy astrometry for some time to come, and the USNO will continue in its efforts to identify and determine the orbital motions of these troublesome objects.

\subsection{OTHER IMAGING}

As discussed above, the RORF program has included a vigorous effort to image the sources of the ICRF. Thus far, there has been no comparable effort for the optical counterparts of these sources. The USNO is currently preparing a proposal to begin imaging the ICRF sources with the Hubble Space Telescope (HST). Although the resolution will be on the order of 10 mas, and therefore not comparable with the RORF data, it will still represent a large improvement over what can be achieved from the ground. Thus, the question of coincidence of emission between the optical and radio counterparts of the ICRF sources still cannot be resolved at the 1 mas level. However, indications of problems are being explored by comparisons of the sources at two different radio wavelengths, generally the X-and K-bands. 
Sources that exhibit a shift at these two frequencies will probably also have optical images that are shifted. This is another area which the USNO will continue pursue.

\section{Links to the Dynamical Frame}

Although the new reference frame will be based on extragalactic objects, there is a long history of observations that have been related to the dynamical frame. These observations still are of importance in some areas such as, for example, the ephemerides of the outer planets. Two USNO programs help deal with this question.

The Washington Fundamental Catalog (Corbin, 1995) will use the existing body of $20^{\text {th }}$ Century data, including Hipparcos to compile a system of 40000 stars on the dynamical system. The catalog will have central epoch positional accuracies of 1 to 2 mas and 0.5 to 1.5 mas/year for the proper motions. Using the results from the first NPOI program and Hipparcos, proper motions on the ICRS system accurate to $0.2 \mathrm{mas} / \mathrm{yr}$ can be achieved for 1000 stars. These then will be used to adjust the WFC system to that of the ICRF. It will then be possible to make accurate reductions to the ICRF over the whole range of the $20^{\text {th }}$ Century.

Another program that can contribute to linking the ICRF and dynamical frames at current epochs is the minor planet program on the USNO Flagstaff $8^{\prime \prime}$ Transit Telescope (Monet, 1995). The program observes near Earth and other asteroids differentially with a high-density set of reference stars. Currently the program accuracy is 300 mas because of the low quality of the reference stars (Guide Star Catalog). However, when the POSS-I/II, becomes available this value will improve considerably. Ultimately, 50 mas should be attainable, and this will provide a valuable check on new versions of the planetary ephemerides by using the asteroids to make the link.

\section{Future Plans}

The USNO is currently a collaborator with Jet Propulsion Laboratory and Naval Research Laboratory in proposing the Fizeau Astrometric Mapping Explorer (FAME). This would be a moderate-cost, 2.5 year satellite mission. The proposal calls for two $10 \times 20 \mathrm{~cm}$ collectors on a $50-\mathrm{cm}$ baseline with a common detector array consisting of eight $4000 \times 1000$ CCDs. The bandpass would be 500 to $900 \mathrm{~nm}$.

The FAME program would produce positions, proper motions and parallaxes of some 10000000 stars to a limiting magnitude of 15 . The precision would range from 0.02 mas at $8^{\text {th }}$ magnitude to 0.80 mas at $15^{\text {th }}$. The corresponding precisions of the proper motion and parallaxes would be 0.02 to $0.80 \mathrm{mas} /$ year and 0.02 to $0.80 \mathrm{mas}$, respectively. 


\section{Conclusion}

The USNO is an active participant in all aspects of establishing an optical reference frame. The RORF and NPOI will make important contributions to establishing and maintaining the axes while the astrographic link program strengthens the radio-optical link. The TAC, SDSS, USNO CCD Astrograph, POSS-I/II and AC projects each will extend the optical frame to higher magnitudes and densities with better accuracies than were attainable before Hipparcos. Finally, the WDS, NPOI and the radio and optical imaging of ICRF sources will address the difficult problems of multiple-image sources, structural changes in sources and coincidence of centers of emission at different wavelengths. The combined effect of all these efforts will be to help establish the best possible optical reference frame as astronomy moves into the $21^{\text {st }}$ Century.

\section{References}

Corbin, T.E.: 1995, in: Astronomical and Astrophysical Objectives of Sub-Milliarcsecond Optical Astrometry, IAU Symposium 166 (E. Hoeg, P.K. Seidelmann, eds), Kluwer, Dordrecht, p.35.

Corbin, T.E. and Urban, S.E.: 1990, in: Inertial Coordinate System on the Sky, IAU Symposium 141 (J.H. Lieske, V.K. Abalakin, eds), Kluwer, Dordrecht, p.433.

Fricke, W., Schwan, H., and Lederle, T.: 1988, "Fifth Fundamental Catalog Part I", Veröff. Astron. Rechen-Inst. No. 32, G. Braun, Karlsruhe.

Fricke, W., Schwan, H., and Corbin, T.: 1991, "Fifth Fundamental Catalog Part II", Veröff. Astron. Rechen-Inst. No. 33, G. Braun, Karlsruhe.

Gunn, J.E.: 1995, Bull. Am. Astron. Soc. $27,875$.

Hutter, D.J., Johnston, K.J., and Mozurkewich, D.: 1995, in: Astronomical and Astrophysical Objectives of Sub-Milliarcsecond Optical Astrometry, IAU Symposium 166 (E. Hoeg, P.K. Seidelmann, eds), Kluwer, Dordrecht, p.23

Hutter, D.J.: 1995, Bull. Am. Astron. Soc. 27, 1452.

Johnston, K.J. et al.: 1995, Astron. J. 110, 880.

Kovalevsky, J. et al.: "The Hipparcos catalog as a realization of the extragalactic reference system", Astron. Astrophys., in preparation.

Monet, A.K.B.: 1995, in: Astronomical and Astrophysical Objectives of Sub-Milliarcsecond Optical Astrometry, IAU Symposium 166 (E. Hoeg, P.K. Seidelmann, eds), Kluwer, Dordrecht, p.405.

Monet, D.G.: 1996, Bull. Am. Astron. Soc. 28, No 3, 905.

Morrison, L.V.: 1995, IAU Working Group on Reference Frames Circular Letter No 1, issued to WGRF members and supporting commissions.

Pier, J.R. and Monet, D.G.: 1993, "Workshop on Databases for Galactic Structure", in: Van Vleck Observatory Contribution No 13 (A.G.D. Philip, B. Hauck, A.R. Upgren, eds), L. Davis Press, Schenectady, p.161.

Urban, S.E. and Corbin T.E. 1996, Astron. Astrophys. 305, 989.

Worley, C.E. and Douglass, G.G.: 1993, "Workshop on Databases for Galactic Structure", Van Vleck Observatory Contribution No 13 (A.G.D. Philip, B. Hauck, A.R. Upgren, eds), L. Davis Press, Schenectady, p.35.

Zacharias, N.: "Astrometric quality of the USNO CCD astrograph", Astron. J., in preparation.

Zacharias, N., de Vegt, C., Winter, L., and Johnston, K.: 1995, Astron. J. 110, 3093.

Zacharias, N., Zacharias, M., Douglass, D., and Wycoff, G.: 1996, Astron. J. 112, 2336. 